\title{
Hierarchical Calcite Crystals with Occlusions of a Simple Polyelectrolyte Mimic Complex Biomineral Structures
}

\author{
Anna S. Schenk, Igor Zlotnikov, Boaz Pokroy, Notburga Gierlinger, Admir Masic, \\ Paul Zaslansky, Andrew N. Fitch, Oskar Paris, Till H. Metzger, Helmut Cölfen, \\ Peter Fratzl,* and Barbara Aichmayer*
}

Biominerals are complex inorganic-organic structures that often show excellent mechanical properties. Here a bio-inspired study of a remarkably simple synthetic system is presented in which only one charged polymer additive (poly(sodium 4-styrenesulfonate)) is able to induce hierarchical structuring of calcite similar to biominerals. The interaction of the negatively charged polymer with the nucleation and growth of the mineral, in particular via selective adsorption to internal and external (001) facets of the calcite lattice, implies structural features from the micrometer down to the nanometer level. The crystals exhibit a distinct rounded morphology and a controlled orientation. Moreover, the polymer molecules are occluded within the crystals with different concentrations in well-defined regions. This leads to the induction of a mesoscale structure based on $100 \mathrm{~nm}$ sized mineral building blocks with granular substructure and rough surface, as well as small modifications of the crystallographic structure. Such a combination of hierarchically organized structural features has previously only been reported for biogenic calcite, which is typically grown in a complex process involving multiple organic additives. It is also shown that the organic occlusions in the calcite-PSS hybrid crystals strongly affect the mechanical performance, as known for some biominerals.

regulate the precipitation of the inorganic phase. ${ }^{[4]}$ Organic molecules were even found to be occluded within biogenic mineral crystals $^{[5-7]}$ thereby inducing anisotropic distortions of the mineral lattice. ${ }^{[8,9]}$

As calcium carbonates are abundant building materials in nature (e.g., in mollusk shells, sea urchins, or corals) and at the same time they have many technological applications (e.g., as filler particles or coatings), this mineral is of great interest in biomimetic materials synthesis. The scope of biologically inspired strategies for the preparation of $\mathrm{CaCO}_{3}$ crystals with defined characteristics ${ }^{[10]}$ includes mineralization in confined environments (insoluble matrices) such as polymeric scaffolds ${ }^{[11]}$ or relatively flexible agarose hydrogel networks ${ }^{[12]}$ (control of morphology and porosity), and template modified crystal growth on two-dimensional substrates such as porous polystyrene films ${ }^{[11]}$ or self-assembled monolayers

\section{Introduction}

Research on biological and bio-inspired minerals has steadily gained importance during the last decades..$^{[1,2]}$ The superior mechanical properties of biominerals are often the result of hierarchical construction ${ }^{[3]}$ employing organic components to

Dr. A. S. Schenk, ${ }^{[+]}$Dr. I. Zlotnikov, Dr. A. Masic, Dr. P. Zaslansky, ${ }^{[++]}$

Dr. T. H. Metzger, Prof. P. Fratzl, Dr. B. Aichmayer,

Max Planck Institute of Colloids and Interfaces

Department of Biomaterials

14424 Potsdam, Germany

E-mail: Peter.Fratzl@mpikg.mpg.de; Barbara.Aichmayer@mpikg.mpg.de

Dr. B. Pokroy

Faculty of Materials Engineering and the Russell Berrie

Nanotechnology Institute, Technion

Israel Institute of Technology

Haifa 32000, Israel

Dr. N. Gierlinger

University of Natural Resources and Life Sciences

Department of Material Sciences and Process Engineering

1190 Vienna, Austria
$(\mathrm{SAMs})^{[13,14]}$ (control of orientation/nucleation and shape). Furthermore, calcium carbonate precipitation can be mediated by soluble additives such as block copolymers ${ }^{[15]}$ or polyelectrolytes $^{[16]}$ including such polymers, which induce the formation of liquid precursor phases ${ }^{[17,18]}$ (control of polymorph, morphology and texture). 
A particularly interesting observation relates to the formation of biological and bio-inspired mesocrystals, where wellaligned nanocrystalline mineral building blocks, often interconnected by a soft organic phase, ${ }^{[16]}$ are supposed to assemble into large organized structures. In spite of the high amount of internal surface area, mesocrystals appear as single crystals by X-ray scattering. Therefore, they can be described as mineral crystals with intracrystalline organic inclusions. For biogenic calcite, the existence of intra-crystalline protein inclusions was to our knowledge first discussed by Towe et al. ${ }^{[19]}$ and experimental evidence was provided more than 20 years ago, when Berman et al. suggested that occluded organics play an important role in the toughening of calcite mineral extracted from sea urchins. ${ }^{[5,6]}$ A simple method for the synthesis of calcite-polymer crystals, where the soluble additive poly(sodium 4-styrenesulfonate) (PSS) was used to grow mesocrystals from an aqueous $\mathrm{CaCl}_{2}$ solution, was reported more recently by Wang et al. ${ }^{[16]}$ In contrast to biogenic calcite crystals, the calcite-PSS mesocrystals have a relatively high content of occluded soluble organics (about $3 \mathrm{wt} \% \mathrm{PSS}^{[16]}$ as compared to less than $0.3 \mathrm{wt} \%$ of intracrystalline soluble proteins in Pinna nobilis). ${ }^{[7]}$

In the present study we analyze the structural principles underlying the occlusion of organic molecules in bio-inspired calcite-PSS crystals. Since neither specialized biopolymers nor confined reaction environments in pre-assembled organic matrices were used in the preparation, the calcite-PSS crystals constitute a model system of striking simplicity. Due to a negative charge on each monomer unit, PSS is assumed to partly resemble the function of soluble proteins involved in the formation of biogenic calcite. Such proteins are known to be rich in negatively charged aspartate residues. ${ }^{[7,20]}$ Moreover, sulfate groups, which are structurally similar to sulfonate, have been identified at the crystal nucleation site of aragonite in mollusc shells. ${ }^{[21]}$ They were also shown to act cooperatively with structured carboxylates on the nucleation of calcite crystals in vitro. ${ }^{[22]}$

We investigated the calcite-PSS hybrid crystals on several length scales ranging from tens of micrometers down to the subnanometer-level by a combination of microscopy, spectroscopy and (synchrotron) X-ray imaging/scattering techniques. Remarkably, we observed a complex hierarchical structure. The structural principles found in the calcite-PSS particles allow for discussing the related physico-chemical mechanisms behind the occlusion of the polymer molecules. Moreover, our results demonstrate that the interaction of forming mineral crystals with only one soluble polymeric additive can generate a highly complex composite comparable in structure and properties to biominerals. These findings are of high relevance for the field of biomineralization and biomimetic materials synthesis. From a broader perspective, we hope to inspire new fabrication methods of hybrid materials with promising properties based on structured multi-length scale organicinorganic interfaces. Besides calcite, different organisms use a variety of about 60 minerals to build hard tissues. ${ }^{[23,24]} \mathrm{New}$ developments, based on the observed principles related to the occlusion of organic molecules and formation of structural hierarchy, could give access to an even wider range of manmade materials far beyond the comparably limited choice of known biominerals.

\section{Results and Discussion}

An overview of the structure of the calcite-PSS particles on 4 levels of hierarchy, characterized by different microscopy and scanning techniques, is given in Figure $\mathbf{1}(\mathrm{a}-\mathrm{h}$ : experimental data; i: schematic illustration). Besides a characteristic outer morphology $(\approx 10 \mu \mathrm{m})$, micrometer sized features within the particles as well as building blocks $(\approx 100 \mathrm{~nm})$ with smaller substructures in the nanometer range are visualized. The experimental findings are described and discussed separately for each level of hierarchy (Sections 2.1 to 2.4). Finally, the results of complementary mechanical measurements are presented in Section 2.5.

\subsection{Morphology and Orientation}

The light microscopy image in Figure 1a indicates that the calcite-PSS crystals were co-oriented on the substrate with respect to their vertical axes. Complementary X-ray diffraction (XRD) measurements (Figure S1, Supporting Information) confirmed a crystal orientation along the crystallographic c-axis possibly due to an adsorption of polymer to the glass surface and subsequent favored nucleation on the (001) plane. It is noteworthy, that unlike studies based on SAMs ${ }^{[25]}$ or templates functionalized with $\beta$-sheet structured carboxylates, ${ }^{[22]}$ an amorphous glass substrate was used in our experiment. Furthermore, upon observation by scanning electron microscopy (SEM), triangular (001)facets were seen on the surface of the crystals (Figure 1b), in agreement with previous studies. ${ }^{[16]}$ According to Wulff's law, ${ }^{[26]}$ such facets are usually not found in pure calcite crystals, since the charged (001)-type lattice planes exhibit high surface energy. Consequently the relative rate of crystal growth perpendicular to these facets is very fast, such that they are usually not exposed to solution. Their exposure on the calcite-PSS crystals can be explained by an electrostatic interaction between the polymer and the mineral leading to a stabilization of (001)-type facets. ${ }^{[16]}$

\subsection{Microstructure}

The 3D internal microstructure of individual crystals was demonstrated by phase contrast-enhanced X-ray microtomography (Figure 1c). With this volume imaging method, contrast is enhanced due to path density differences arising from compositional and/or structural inhomogeneities..$^{[27,28]}$ In addition to the characteristic outer morphology, concealed micrometersized inner surfaces were observed. Confocal scanning Raman microscopy revealed the existence of two distinct domains with different polymer contents (Figure 1d): a central domain is PSS enriched by a factor of 2.5 compared with the outer part (see also Figure S2,S3, Supporting Information). Etching by placement of the crystals in de-ionized water or hydrochloric acid (Figure S4, Supporting Information) provided further evidence of the presence of structurally and chemically different domains, on the $\mu \mathrm{m}$-lengthscale.

\subsection{Structure at the Mesoscale and Intracrystalline Occlusions}

At a lower hierarchical level corresponding to the mesocrystalline structure, the exposed (001)-facets are composed of 


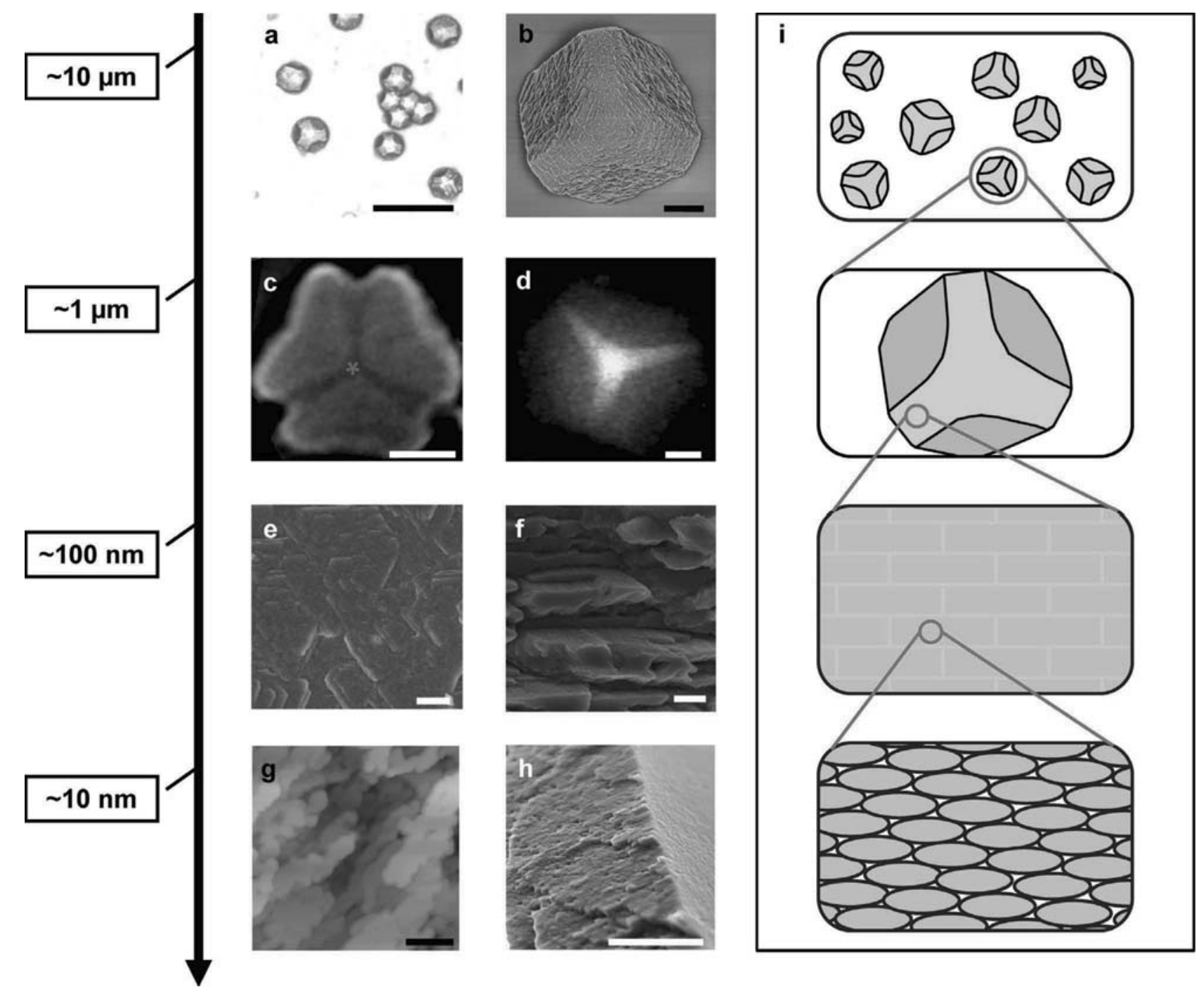

Figure 1. Structural organization on several length scales. The shown calcite-PSS crystals were grown from a solution containing the following reactant concentrations: $\left[\mathrm{Ca}^{2+}\right]=1.25 \mathrm{~mm}$ and $[\mathrm{PSS}]=0.5 \mathrm{~g} / \mathrm{L}$. a) Light microscopy illustrates the co-orientation of the crystals on the glass substrate (scale bar $=100 \mu \mathrm{m}$ ), while b) SEM gives an overview of the overall morphology of an individual bio-inspired crystal (scale bar $=10 \mu \mathrm{m}$ ). The displayed crystal exhibits rounded corners and exposes the usually highly charged (001)-facet. c) Phase contrast enhanced synchrotron X-ray tomography visualizes the 3D structure of an individual calcite-PSS crystal. The image shown represents a $2 \mathrm{D}$ slice cut perpendicular to the $(001)$-facet $(\mathrm{scale}$ bar $=20 \mu \mathrm{m})$. A clear contrast difference between a region with a three-fold symmetry in the particle center (red asterisk) and the outer regions is revealed. d) An investigation of the chemical composition was performed by confocal Raman microscopy. The intensity distributions of a spectral line assigned to PSS (symmetric stretching mode of the sulfonate group, wavenumber $=1135 \mathrm{~cm}^{-1}$ ) is mapped (scale bars $=10 \mu \mathrm{m}$ ) uncovering an accumulation of the organic molecules in the particle center. e) An SEM image (top view) of the (001)-facet shows an assembly of well-aligned mineral building blocks corresponding to a mesocrystalline structure $(\mathrm{scale} b a r=1 \mu \mathrm{m})$. In $(\mathrm{f})$ a side view of the mineral platelets is shown $(\mathrm{scale}$ bar $=100 \mathrm{~nm})$. g) AFM studies of the crystal surface $(s c a l e ~ b a r=200 \mathrm{~nm}$ ) reveal a granular substructure in the aligned mineral building blocks. h) Very small mineral units of only tens of nanometers in thickness are also observed on a fracture surface (scale bar $=1 \mu \mathrm{m}$ ) by high resolution SEM. i) Schematic illustration of the hierarchical structure. The mineral is drawn in gray, whereas the intracrystalline organic component is visualized in orange color.

well-aligned plate-like building blocks (see Figure 1e (top view) and $\mathrm{f}$ (side view) as well as Figure $\mathrm{S} 5$ in the Supporting Information). Remarkably, atomic force microscopy (AFM) revealed that these $\approx 200 \mathrm{~nm}$ thick platelets consist of smaller subunits measuring $\approx 30 \mathrm{~nm}$ (Figure 1g). Grains were observed not only on the external surfaces, but also on internal fracture surfaces of the hybrid crystals (Figure 1h). Hence, fracture of the calcitePSS crystals did not induce cleavage along the (104) plane, but resulted in rough fracture surfaces. The non-facetted, roundish grains are reminiscent of structural features akin to those, which constitute the initially deposited phase in the growth of the prismatic layer of the mollusc shell Atrina rigida. ${ }^{[20]}$

The preferential orientation of the nanostructure with respect to the mineral lattice was studied in more detail by means of simultaneous small- and wide-angle X-ray scattering measurements (SAXS/WAXS) on single crystals ( $\mu$-Spot beamline, BESSY II synchrotron storage-ring, Helmholtz-Zentrum Berlin für Materialien und Energie, Germany). ${ }^{[29]}$ 
The calcite-PSS particles showed wide-angle diffraction spots (Figure 2a) typical of single crystals as well as a strong anisotropic SAXS signal (Figure 2b) arising from electron density differences on the nanometer-scale. These density differences most likely reflect the internal organic-inorganic interfaces. The nanostructural motifs were found to be oriented parallel to the neutral (104)-type and the charged (001)-type lattice planes (Figure 2c,d). While the alignment with the low energy (104)type planes hints to a faceting of the mineral building blocks along the typical calcite cleavage planes, the correlation to the (006)-Bragg reflections can be explained by electrostatic adhesion of occluded negatively charged PSS molecules, similar as for the external (001) facets.

A congruence between the external crystal morphology and the shape of organic occlusions was recently also reported by Kim et al. for calcite incorporating $20 \mathrm{~nm}$-sized block copolymer micelles. ${ }^{[30]}$ However, exclusively (104)-type facets were identified in this study by means of transmission electron microscopy (TEM). In calcite crystals with occluded agarose hydrogels, both low and high energy facets were found at the fibre/crystal interface. ${ }^{[12]}$ In contrast to the block copolymer micelles and the here investigated PSS molecules, where the preferential adsorption to specific lattice planes determines the interfaces, the facets in the calcite/agarose system presumably result from a physical entrapment where the interfaces follow the curvature of the hydrogel network. ${ }^{[12]}$

An estimate of the mean size of the nanoscopic polymer inclusions separating the mineral blocks was obtained by analyzing a powder sample. The orientation-averaged SAXS profile plotted as a function of the length of the scattering vector Q (Figure 3a, filled circles) can be divided into three regimes (I, II, III). At very low values of $\mathrm{Q}\left(\mathrm{Q}<0.25 \mathrm{~nm}^{-1}\right.$, Figure 3a, region I), the signal shows a linear decay on the log-log scale (with a power law exponent of roughly -4), which corresponds to scattering from a large surface area, presumably originating from the external surfaces of the calcite-PSS crystals (outer surfaces of the whole crystals). Evidence for this assumption was provided by contrast variation (Figure S6, Supporting Information). The strong anisotropy of the SAXS signal seen in single crystal measurements (Figure 2) in combination with the plate-like shape of the mineral building blocks (Figure 1e,f) suggests that the organic inclusions (or partially polymer-filled pores) between the mineral blocks are also platelet-shaped.

A Guinier analysis of region II $\left(0.4 \mathrm{~nm}^{-1}<\mathrm{Q}<1.2 \mathrm{~nm}^{-1}\right.$, surface contribution of region I not taken into account, since this part of the signal was negligible at higher values of $\mathrm{Q}$ ), assuming diluted plate-like inhomogeneities ${ }^{[31]}$ (i.e., $d>D$, where $\mathrm{D}=$ thickness of platelet-shaped organic occlusions and $\mathrm{d}=$ interplatelet distance), yielded a thickness of $\mathrm{D}=$ $2.7 \pm 0.03 \mathrm{~nm}$ (see also Figure S7, Supporting Information). This value is lower than the mean radius of gyration $R_{g}=6.3$ $\mathrm{nm}$ determined from a Debye-fit ${ }^{[32]}$ of the scattering profile recorded for globular polymer aggregates in an aqueous $\mathrm{Ca}^{2+}$. solution (Figure S8, Supporting Information), but is higher than the diameter of an individual polymer chain $(\approx 1.5 \mathrm{~nm})$. We thus propose that a more elongated conformation of the intracrystalline polymer molecules exists, as compared with the structure in solution.
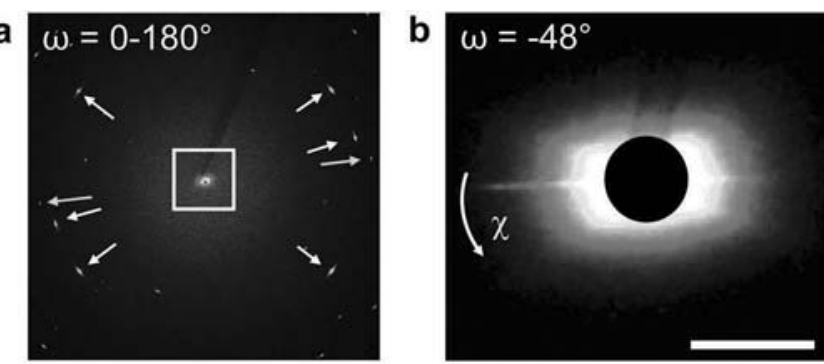

C

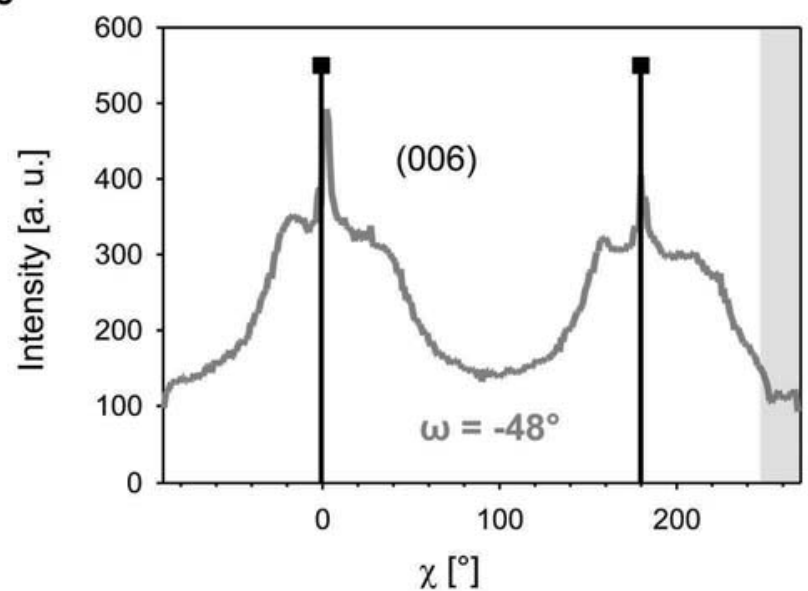

d

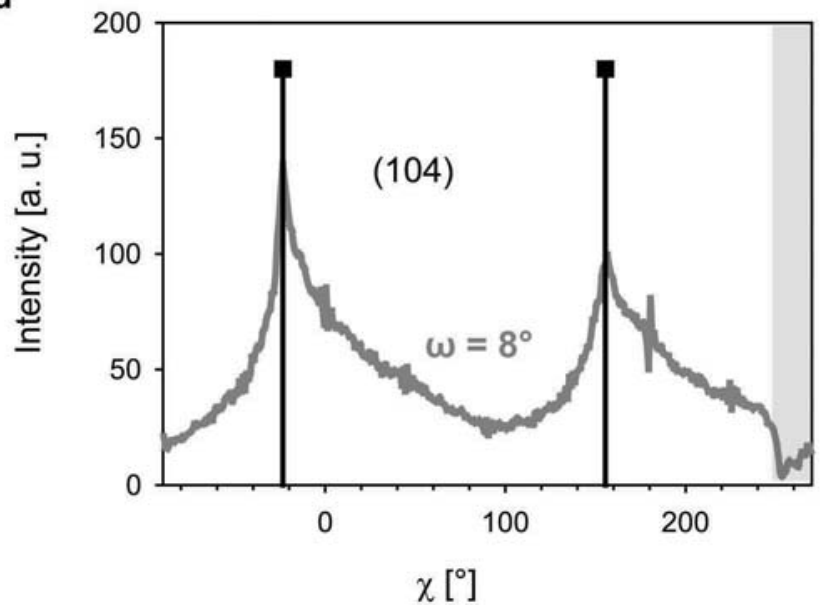

Figure 2. Microbeam small- and wide-angle X-ray scattering of single calcite-PSS crystals. a) The 2D-SAXS/WAXS scattering pattern recorded during a $180^{\circ}$ rotation of an individual calcite-PSS crystal. The anisotropic SAXS signal in the center of the image (framed in white) indicates the presence of a pronounced nanostructure in the hybrid crystal. Diffraction spots, as they are typical for single crystals, are observed in the wideangle region (white arrows: (104)- type, yellow arrows: (006)-type Bragg reflections). The SAXS pattern depicted in (b) was taken at a fixed rotation angle $(\omega)$. The intensity streak points to the crystallographic orientation of the (006)-type lattice planes of calcite (scale bar $=1 \mathrm{~nm}^{-1}$ ). The azimuthal angle $\chi$ is illustrated by the white arrow. c,d) Profiles of the intensity of the scattering signal vs. the azimuthal angle $\chi$ are plotted for two distinct sample rotation angles $\omega$, where maxima appear in the anisotropy of the SAXS signal. The positions of the measured (006)- and (104)- diffraction spots (black squares) correspond well to the pronounced maxima in the anisotropy of the SAXS signal (gray line). The angular range marked with a light blue bar was affected by a glass capillary holding the beamstop. 


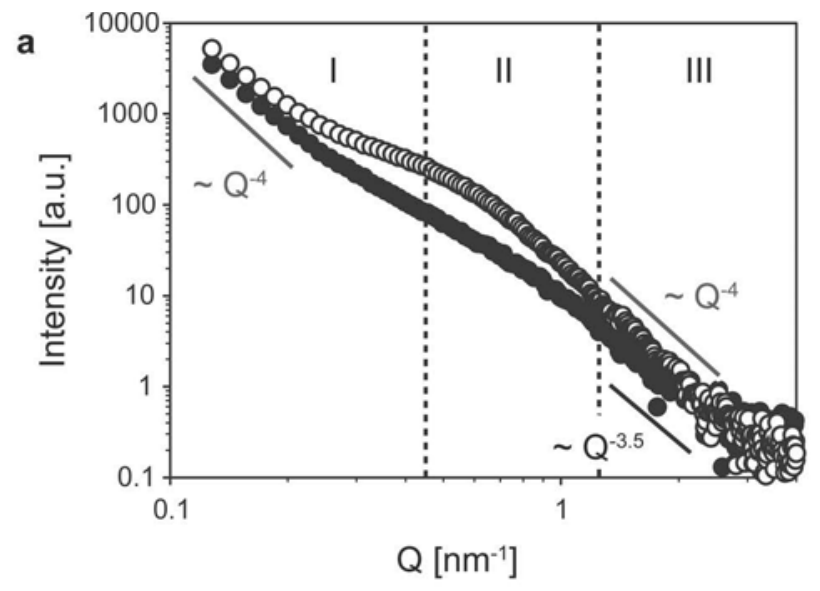

b

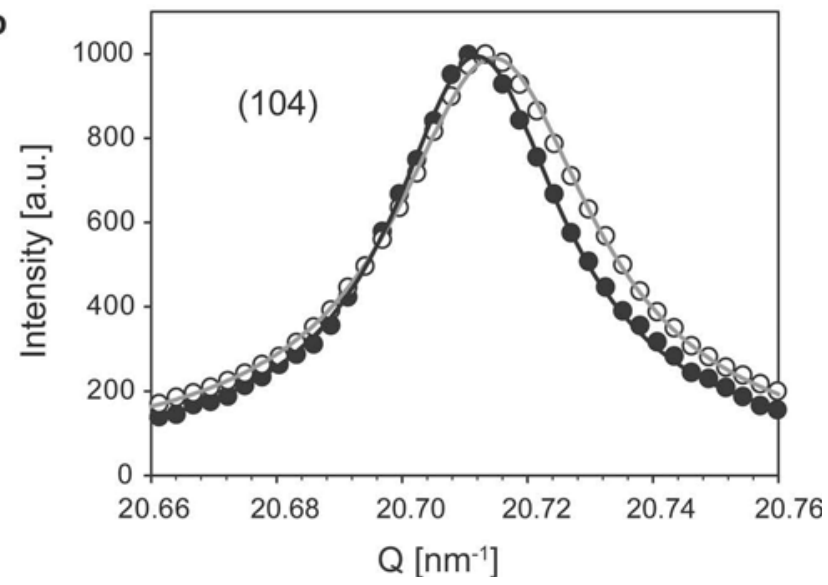

Figure 3. Small-angle $\mathrm{X}$-ray scattering and high resolution $\mathrm{X}$-ray diffraction of powder samples. a) Small-angle scattering profile of bio-inspired crystals (filled circles) fitted with a power law function at large values of the scattering vector $Q$. The dashed lines indicate three different regimes. At very low $\mathrm{Q}$ (region I) the signal shows a steep decay, originating from the external surfaces of the calcite-PSS crystals. In the intermediate $\mathrm{Q}$ range (II) the signal of the nanoscopic intracrystalline inclusions becomes more dominant. At large Q (area III) the curve follows a steep decay proportional to $\mathrm{Q}^{-3.5}$ (black line), deviating from Porod's law. The profile recorded subsequent to annealing at $400{ }^{\circ} \mathrm{C}$ (open circles) shows a characteristic intensity decay $\mathrm{I}(\mathrm{Q}) \approx \mathrm{Q}^{-4}$ at large $\mathrm{Q}$ (gray line) indicating a heat-induced smoothening of initially rough interfaces. b) XRD peak profiles of the (104)-reflection of bio-inspired crystals recorded before (filled circles) and after annealing at $400{ }^{\circ} \mathrm{C}$ (open circles). A limited angular range is depicted and the error bars are smaller than the symbols. The heat-treatment results in a small lattice shift to a lower d-spacing as well as a broadening of the diffraction line.

In the third $\mathrm{Q}$ range (Figure 3a, region III, $1 \mathrm{~nm}^{-1}<\mathrm{Q}<$ $2 \mathrm{~nm}^{-1}$ ) an exponent of $-3.5 \pm 0.2$ was extracted from a power law fit of the SAXS profile. For two homogenous phases separated by a sharp interface an exponent of -4 is expected, according to Porod's law. ${ }^{[33]}$ This deviation from the $\mathrm{Q}^{-4}$ dependence ${ }^{[34]}$ suggests that the surface of the mineral building blocks is rough. Annealing for 1 hour at a temperature of $400{ }^{\circ} \mathrm{C}$ (Figure 3a, open circles) caused a change of this slope to the expected slope of -4 (Porod's law) indicating a smoothening of the rough surfaces. A similar smoothening effect of initially rough organic-inorganic interfaces after a mild heat treatment had previously also been observed for biogenic calcite with occluded organic molecules. ${ }^{[35]}$

\subsection{Lattice Structure and Size of Coherent Domains in the Mineral Phase}

At the Ångstrom lengthscale, corresponding to the calcite lattice structure and the crystallite size of the building blocks, the calcitePSS crystals were investigated by means of high resolution powder X-ray diffraction (beam line ID31, ESRF, Grenoble, France). ${ }^{[36]}$

From the broadening of the (104) diffraction peak shown in Figure 3b (black circles), a mean domain size of $214 \pm 4 \mathrm{~nm}$ perpendicular to the (104)-type planes was estimated, based on the Scherrer equation (for details on the treatment of size effects and microstrain fluctuations see Experimental Section). We found coherent domains sized $155 \pm 5 \mathrm{~nm}$ and $147 \pm 2 \mathrm{~nm}$ from the broadening of the (110) and (006) peaks, respectively (Figure S9, Supporting Information). These dimensions are in good agreement with the thickness of the plate-like mineral blocks as visible in the SEM (Figure 1f). The size of the granular subunits (Figure 1g,h), however, was estimated to be in the range of $30 \mathrm{~nm}$ by means of AFM and SEM and therefore substantially smaller than the coherently scattering domains. Our conclusion is that these grains are arranged within a perfect crystallographic register. A possible explanation for this observation, which was also suggested for biogenic calcite, ${ }^{[22]}$ would be the formation of a granular amorphous precursor phase that crystallizes after the grains have fused together.

For biogenic calcite Pokroy et al. reported a pronounced broadening of the diffraction peaks as well as anisotropic lattice distortions after mild annealing treatments. ${ }^{[9,36]}$ Similarly, we compared the diffraction lines of the bio-inspired calcite-PSS crystals with those measured for an annealed sample $\left(400{ }^{\circ} \mathrm{C}\right.$, $1 \mathrm{~h}$, in air atmosphere). The chosen annealing temperature can be regarded as relatively mild for the calcite crystal structure (low diffusion), but allows rearrangements of the occluded polymers. The diffraction peak maxima (open circles, Figure $3 \mathrm{~b}$ ) were only very slightly shifted to lower lattice spacings $\left(\Delta \mathrm{d} / \mathrm{d}=-1.3 \times 10^{-4}\right.$ for the (104) peak, $\Delta \mathrm{d} / \mathrm{d}=-1.4 \times 10^{-4}$ for the (110) peak) when compared to the untreated sample (filled circles). Temperature-induced lattice rearrangements previously observed in biogenic calcite ${ }^{[9]}$ were significantly bigger than those that we identified in our biomimetic specimen. However, after annealing, a noticeable broadening of the diffraction lines was observed for calcite-PSS. Similar to biogenic calcite crystals, ${ }^{[37]}$ the size of the coherently scattering domains decreased (178 $\pm 1 \mathrm{~nm}$ and $142 \pm 5 \mathrm{~nm}$ for (104) and (110), respectively), when the occluded organic molecules were rearranged due to heat treatment. In agreement with the relatively mild annealing temperature, we did not observe fusing of the subunits, which would result in an increase of the measured size. This finding strengthens the notion that a strong interaction exists between the PSS molecules and the calcite mineral lattice.

\subsection{Mechanical Properties}

Finally, motivated by the astonishing mechanical behaviour of biominerals, we studied the mechanical properties of the 
calcite-PSS hybrid crystals by means of nanoindentation on embedded and finely polished samples. The results strongly reflected the compositional differences within the crystals. A mean hardness of $\mathrm{H}=2.0 \pm 0.25 \mathrm{GPa}$ and a reduced modulus of $E_{r}=26.0 \pm 3.4 \mathrm{GPa}$ were measured in the central part of the crystals, which was found to exhibit a higher polymer content, compared to $\mathrm{H}=2.7 \pm 0.4 \mathrm{GPa}$, and $\mathrm{E}_{\mathrm{r}}=40.8 \pm 5.5 \mathrm{GPa}$ in the outer polymer-deficient regions of the crystals. Biogenic calcite prisms extracted from Pinna nobilis shells showed a comparatively higher hardness and reduced modulus $(\mathrm{H}=3.5 \pm$ $0.25 \mathrm{GPa}, \mathrm{E}_{\mathrm{r}}=56.3 \pm 2.1 \mathrm{GPa}$ ) when analyzed under the same experimental conditions. Biogenic calcite was previously found to exhibit a higher hardness than pure calcite, but often a solid solution of $\mathrm{Mg}^{2+}$ is involved in the hardening mechanisms, e.g., in the case of the sea urchin tooth. ${ }^{[38]}$ Hence, we chose calcite prisms extracted from mollusk shells, which have a relatively low magnesium content ( 2 at.- $\%$ in Pinna nobilis), ${ }^{[9]}$ as a biogenic sample for comparison. The difference between the Pinna nobilis prisms and the calcite-PSS crystals can be assigned to both a lower content of organic phase in the biogenic material and a different microstructure. For the calcite-PSS crystals, assuming a similar microstructure in the outer and inner regions of the crystal, we can conclude that the lower hardness and modulus in the central part result from the higher polymer content. Based on the change in hardness and on the observed rough fracture surfaces (see Figure 1h), we hypothesize that in analogy to biominerals with intra-crystalline structures, ${ }^{[39]}$ the high amount of organic-inorganic interfaces leads to increased fracture toughness of the biomimetic crystals, although further work is needed to confirm this.

\section{Conclusions}

In summary, our results demonstrate that the interaction of forming mineral crystals with only one soluble polymeric additive can generate highly complex composites with a hierarchical structure $^{[40]}$ and oriented, rough, organic-inorganic interfaces ${ }^{[35]}$ comparable to biominerals. We observed controlled nucleation from the crystallographic (001) plane, the formation of external (001) facets and the intra-crystalline occlusion of PSS along (001) interfaces, which is consistent with the mesocrystal model ${ }^{[16]}$ previously proposed. Surprisingly, the well-oriented intra-crystalline interfaces exhibit a fractal-like roughness which disappears upon annealing at $400^{\circ} \mathrm{C}$. Fractal-like protein-calcite interfaces were most recently also found in biogenic crystals from mollusc shells. ${ }^{[35]}$ This roughness suggests a close interaction between organic and inorganic components, most likely via electrostatic forces between the charged calcite (001) planes and the sulfonate groups of the polyelectrolyte occlusions. The overall stiffness of the material is reduced dependent on the content of occluded polymer molecules. We can only speculate that the calcite-PSS crystals might be formed in a two-step process, in which the polymer concentration is depleted in the later stages, thus giving rise to a reduced content of occluded organics in the outer domains of the particles. Furthermore, the organic occlusions are likely responsible to prevent macroscopic cleavage along the (104) plane, which was not observed here, similarly to biogenic calcite, ${ }^{[6]}$ thereby reducing its intrinsic brittleness. Thus, this synthetic approach proves that, by using only one charged polymer additive, complex biomineral-like structures with promising mechanical properties can be obtained much easier than it was previously assumed.

\section{Experimental Section}

Chemicals: The following commercially available chemicals were purchased and applied in the syntheses without further purification: $\mathrm{CaCl}_{2} \cdot 2 \mathrm{H}_{2} \mathrm{O}$ (p.A.; Sigma-Aldrich), poly(sodium 4-styrenesulfonate) $\left(\mathrm{M}_{\mathrm{w}}=70000 \mathrm{~g} / \mathrm{mol}\right.$; Aldrich), $\mathrm{NaOH}$ platelets ( $\geq 98 \%$; Fluka), $\left(\mathrm{NH}_{4}\right)_{2} \mathrm{CO}_{3}$ (Fluka). For the preparation of the reactant solutions double-distilled and deionized (Milli-Q) water was used.

Synthesis of the Bio-Inspired Crystals: The synthesis of biomimetic calcite-PSS crystals $^{[16]}$ was performed according to a gas diffusion technique. Crystal growth took place on glass slides, which were each placed on the bottom of a glass vial. All glassware was first sonicated in ethanol, subsequently rinsed with distilled water, then sonicated in saturated $\mathrm{NaOH}$ solution, rinsed with double-distilled water and finally dried at room temperature. Stock solutions of $\mathrm{CaCl}_{2}$ and PSS were produced in double-distilled water, which had been bubbled with nitrogen overnight. From these stock solutions the crystallization solution containing $\mathrm{Ca}^{2+}(1.25 \mathrm{~mm})$ and PSS $(0.5 \mathrm{~g} / \mathrm{L})$ was freshly prepared. The glass vials containing a glass slide on the bottom and $5 \mathrm{~mL}$ of the reactant solution were covered with Parafilm, which was subsequently punched with three needle holes, and placed in a desiccator. As a source of $\mathrm{CO}_{2}$ and ammonia, crushed ammonium carbonate was filled into two additional glass vials, equally covered with Parafilm, punched with three needle holes and placed at the bottom of the desiccator. After five days the samples were removed from the reaction solutions. The glass slides carrying the calcite crystals were rinsed in distilled water as well as acetone and dried at room temperature.

Dialysis of the Polymer (PSS) Solution: In order to produce a sample of biomimetic calcite crystals free of the sodium ions introduced by the polyelectrolyte, an aqueous solution of poly(sodium 4-styrenesulfonate) was dialyzed against deionized water utilizing a dialysis tubing purchased from Spectra/Por (Cellulose/Ester, MWCO: 3500 Dalton, diameter: $29 \mathrm{~mm}$ ). The dialysis was run for four days and the surrounding water was exchanged twice a day. After purification the polymer was applied in a gas diffusion reaction as described above.

Light Microscopy: Light microscopy was performed by the use of a Leica microscope (DM RXA2), which was operated in the transmission mode.

$X$-Ray Microtomography: Individual crystals were glued to the tip of tapered glass capillaries, fixed on a sample-holder and mounted upright in the microtomography stage of the BAMline imaging setup at the BESSY II storage ring (Helmholtz-Zentrum Berlin für Materialien und Energie). For each dataset, 400 radiographs were recorded at angular increments of 0.45 using a partially coherent and quasi monochromatic beam of $30 \mathrm{keV}$, setting a sample-to-detector distance of $300 \mathrm{~mm}$ and using imaging optics and lenses with an effective pixel size of $0.87 \mu \mathrm{m}$. The projection images were normalized and reconstructed using Octopus V8.1 (XrayLAB, Chent University, Belgium). Thereafter, the crystals were visualized (Amira 4.1, Visage Imaging $\mathrm{GmbH}$, Germany) and the data was rotated, cropped, sliced and analyzed to reveal internal structures and edge-enhanced features.

Confocal Raman Microscopy: The composition on the micrometer level was studied with a confocal Raman microscope (CRM300, WITec, Ulm, Germany) equipped with a piezo-scanner (P-500, Physik Instrumente, Karlsruhe, Germany), a 100× (0.9 NA) objective, and a $532 \mathrm{~nm} \mathrm{Nd:YAC}$ laser. An air-cooled CCD detector (DU401-BV, Andor, UK) placed behind the spectrometer (UHTS 300, WITec, Germany) was used for data collection. The laser power was adjusted appropriately to have a sufficient signal-to-noise ratio and to avoid sample degradation. The ScanCtrlSpectroscopyPlus software (version 1.38, WITec, Ulm, Germany) was used for measurement and WITec Project Plus (version 2.02, WITec, 
Ulm, Germany) for spectra processing. Raman imaging was performed by scanning the sample at the chosen depth in spatial increments of $0.5 \mu \mathrm{m}$ with an integration time of $0.5 \mathrm{~s} / \mathrm{spectrum}$. The spectra obtained were slightly smoothened using the 5-point Savitzky-Golay filter (4th order polynomial). The WITec project basis analysis function was applied to map the distribution of the individual components. The underlying algorithm fits each spectrum of the multigraph data object with a linear combination of the basis single spectra (measured for control calcite and polymer) using the least squares method. To solve the problem of different background in various parts of the sample, the first derivative of both the multi-graph data object and the basis spectra was calculated.

In Figure $1 d$ the weighting factors obtained by fitting of the PSS spectrum are shown. Those bands assigned to the symmetric stretching mode of the sulfonate group in PSS (wavenumber $=1135 \mathrm{~cm}^{-1}$ ) and the symmetric stretching mode of the carbonate ion in the mineral (wavenumber $=1090 \mathrm{~cm}^{-1}$ ) were integrated for imaging the polymer and calcite distribution, respectively. The calculation of the relative amounts of PSS in different parts of the crystals was based on the area underneath the vibration band assigned to the symmetric stretching mode of the sulfonate group at $1135 \mathrm{~cm}^{-1}$ (after normalization for the carbonate content).

Environmental Scanning Electron Microscopy/High Resolution Electron Microscopy: For sample topography imaging an environmental scanning electron microscope (Quanta FEG 600, FEI, The Netherlands) was applied. The instrument was generally operated in the low vacuum mode, so that sputtering of the samples was not necessary. A large field detector (LFD) was used for signal collection and an acceleration voltage of $5 \mathrm{kV}$ was chosen for recording the images. In order to investigate fracture surfaces of the samples in more detail high-resolution electron microscopy (HRSEM) was performed. The experiments were carried out on a scanning electron microscope (JSM-7500F, JEOL, Japan) operated at an acceleration voltage of $5 \mathrm{kV}$ in high vacuum. The specimens were coated with a very thin layer of carbon in order to avoid charging effects.

Atomic Force Microscopy: Atomic force microscopy (AFM) investigations have been performed on untreated crystals using a Nanoscope IIla instrument (Dimension 3100, Veeco instruments, Santa Barbara, USA). All images were collected at room temperature in the tapping mode utilizing an Al-coated silicon tip (purchased from NanoWorld) exhibiting a typical resonance frequency of $285 \mathrm{kHz}$ and a force constant of $42 \mathrm{~N} / \mathrm{m}$.

Microbeam Small- and Wide-Angle X-Ray Scattering: Simultanous SAXS/WAXS investigations of single crystals were performed at the $\mu$-Spot beamline of the synchrotron facility BESSY II (HelmholtzZentrum für Materialien und Energie, Berlin). ${ }^{[29]}$ Radiation with an energy of $12.4 \mathrm{keV}$ was provided by a Si 111 monochromator. The diameter of the X-ray beam was defined by a $30 \mu \mathrm{m}$ pinhole. For the measurements the crystals were glued on glass capillary tips, which were subsequently fixed on a goniometer allowing for an alignment of the sample in the beam. The crystals were rotated through $180^{\circ}$ in steps of $2^{\circ}$ (rotation angle $\omega$ ) while after each step a scattering pattern was recorded. Additionally, an averaged SAXS/WAXS profile was measured continuously during a $180^{\circ}$ rotation. A MarMosaic 225 detector (Mar USA, Evanston, USA) consisting of nine independent 16-bit CCD chips connected to a phosphor screen of $225 \mathrm{~mm}$ edge length was used for data collection.

The 2D scattering patterns were transformed into 1-dimensional profiles of the scattering intensity vs. the azimuthal angle $\chi$ by integration using the software Fit2D (A. Hammersley, ESRF, Grenoble, France). The modulus of the scattering vector was defined as $Q=$ $4 \pi \sin (\theta) / \lambda$, where $\lambda$ denotes the wavelength and $2 \theta$ is the scattering angle. While the small-angle signal was radially averaged in a Q-range of $0.4 \mathrm{~nm}^{-1}<\mathrm{Q}<4.5 \mathrm{~nm}^{-1}$, an interval of $\Delta \mathrm{Q}=3 \mathrm{~nm}^{-1}$ centred at the position exhibiting the maximum intensity was typically chosen for the integration of the wide-angle diffraction spots. To allow a common representation of the SAXS and the WAXS signals, only the azimuthal positions of the wide-angle spots corresponding to the (006)-reflection and the (104)-reflection are indicated in Figure 2, while the respective intensities were scaled arbitrarily. For calibration of the beam center as well as the sample-detector distance and other geometrical effects a standard powder consisting of alpha-Quartz (NIST Standard Reference Material 1878a) was used. In order to identify orientation correlations between the mineral lattice and the occluded organics (generating the SAXS signal), we compared the frames showing the most pronounced anisotropy in the small-angle scattering signal (occurring at rotation angles $\omega_{\text {SAXS }}$ ) with those frames containing the correspondingly oriented wide-angle peaks. Due to geometrical considerations, the WAXS peaks were excited at rotation angles of $\omega=\omega_{\text {SAXS }} \pm 10^{\circ}$ as compared to the anisotropic SAXS signal.

Small-Angle X-Ray Scattering (Laboratory Instrument): A Nanostar instrument (Bruker AXS, Karlsruhe, Germany) was used for smallangle X-ray scattering (SAXS) measurements of powder samples. The X-ray generator of the device was operated at $40 \mathrm{kV}$ and $35 \mathrm{~mA}$. Cu- $\mathrm{K}_{\alpha}$ radiation with a wavelength of $\lambda=0.154 \mathrm{~nm}$ was selected by a parabolically bent, graded multilayer (Göbel mirror) resulting in a parallel and monochromatic beam. For the scattering experiments the instrument was kept under vacuum in order to prevent air scattering. For the collection of two-dimensional scattering patterns a single photon counting area detector (HiStar, Bruker AXS) was applied. All measurements were consecutively carried out at two different settings of the sample-detector distance being $26 \mathrm{~cm}$ and $105 \mathrm{~cm}$, respectively. This approach allowed for covering the overlapping Q-ranges of $0.1 \mathrm{~nm}^{-1}<$ $\mathrm{Q}<2 \mathrm{~nm}^{-1}$ in the longer and $0.5 \mathrm{~nm}^{-1}<\mathrm{Q}<7 \mathrm{~nm}^{-1}$ in the shorter configuration, where $\mathrm{Q}$ represents the modulus of the scattering vector. The powdered (bio-inspired crystals) or in water dissolved (poly(sodium 4-styrenesulfonate)) samples were filled into borosilicate capillaries (diameter $=1 \mathrm{~mm}$ or $1.5 \mathrm{~mm}$ ) and fixed on a metal sample holder, which was mounted on a movable $x-y$ stage. The scattering patterns were taken at three different points along the longitudinal axis of the capillary.

The resulting $2 \mathrm{D}$ detector images were orientation averaged in order to obtain one-dimensional scattering profiles, which were subsequently corrected for instrument-related background and transmission. The normalized scattering signal of the sample container, which is in this case represented by an empty glass capillary, was subtracted. Afterwards, the corresponding curves measured at $105 \mathrm{~cm}$ and $26 \mathrm{~cm}$ were merged. For this purpose, a linear relationship between the intensities in the overlap region was assumed: $I_{26 \mathrm{~cm}}=a l_{105 \mathrm{~cm}}+b(\mathrm{a}, \mathrm{b}$ constant, $\mathrm{b}=0$ after correction for instrument-related background). For the powderous sample of bio-inspired crystals the power-law dependence at large values of the scattering vector was analyzed $\left(I(Q)=I_{0} Q^{-p}+C\right)$ and a constant sample-related background $C$ was subtracted. Finally, the processed scattering curve was subjected to a Guinier evaluation valid for platelike features. Therefore, the function $I(Q)=\left(\pi / Q^{2}\right) \exp \left(-D^{2} Q^{2} / 12\right)$, with $D$ being the platelet thickness, was fitted to the data in the Q-range $0.4 \mathrm{~nm}^{-1}<\mathrm{Q}<1.2 \mathrm{~nm}^{-1}$. The scattering curve obtained for the polymer in aqueous solution, on the contrary, was fitted by the Debye function, ${ }^{[32]}$ which is valid for flexible polymers with Gaussian statistics $(I(Q) \propto$ $2[\exp (-u)+u-1] / u^{2}$ with $u=\left\langle R_{g}{ }^{2}\right\rangle Q^{2}, R_{g}$ being the radius of gyration). In this context, $R_{g}$ can be interpreted as a measure for the mean dimension of the polymer aggregates.

High Resolution X-Ray Diffraction: High-resolution X-ray powder diffraction was performed at beamline ID31 of the European Synchrotron Radiation Facility (ESRF, Grenoble France), ${ }^{[36]}$ which uses a liquidnitrogen-cooled double-crystal silicon 111 monochromator to select the wavelength required from the radiation provided by the undulators in the synchrotron storage ring. For the collection of the diffracted intensity a bank of nine detectors, each preceded by a silicon 111 analyzer crystal, was used. A silicon standard sample (NIST 640c) purchased from the National Institute of Standards and Technology (NIST, Gaithersburg, MD, USA) was measured to calibrate the wavelength. The powders were contained in $1 \mathrm{~mm}$ diameter borosilicate glass capillaries, which were rotated during the investigations in order to improve the powder average and reduce preferred-orientation effects. For the evaluation of line broadening related to the instrument a standard $\mathrm{LaB}_{6}$ sample with a defined grain size in the range of micrometers (NIST Standard Reference Material 660a) was measured. Since the polyelectrolyte was purchased as a sodium salt, the PSS solution applied for the preparation 
of the bio-inspired crystals had to be dialyzed against water prior to the experiment in order to eliminate sodium ions and to exclude their effects on the calcite lattice.

The crucial factor for the precision of the used experimental setup, when comparing different, subsequently measured diffractograms, is the stability, which we monitored by repeated measurements of the standard powder of silicon, yielding a maximum difference of $\Delta d / d=4 \times 10^{-5}$. Generally, we observed a broad component of the peak underneath a much more intense narrower one, indicating a second structura component within the crystals or a contribution of nanoparticles sedimented from the crystallization solution. The peak positions, however, were predominantly determined by the narrower component. Accordingly, the peaks were fitted with a sum of two Lorentzians and the values we report with regard to positional shifts are based on the maximum of the narrower component. Within the given precision, an evaluation of only the narrow components of the peaks gives the same values as found for the sum of the two components. The peaks were fitted after subtraction of a linear background. In order to allow a better comparability, the diffraction lines depicted in Figures $3 \mathrm{~b}$ and $\mathrm{S} 9$ were normalized to a maximum intensity of 1000 .

For the analysis of the Scherrer size, the peaks were assumed to be fully Lorentzian in shape. This approach does not allow an explicit separation of size- and strain-related line broadening. However, an analysis of higher order peaks [( $\left.\begin{array}{lll}2 & 0 & 8\end{array}\right),\left(\begin{array}{lll}0 & 0 & 12\end{array}\right)$ and $\left.\left(\begin{array}{lll}2 & 2 & 0\end{array}\right)\right]$ yielded no additional broadening, which indicates that the line profile is mainly dominated by the size of the crystallites. Moreover, strain effects, which are supposed to appear as a contribution of a Gaussian-type component in the profile, would even further reduce the line broadening attributable to the limited size of the coherently scattering domains. A smaller value for the size-related broadening, in turn, corresponds to an even bigger dimension of the coherent entities. Hence, the here-presented values reflect lower limits.

Embedding, Polishing and Nanoindentation: For nanoindentation, specimens with a completely flat surface were required. For this purpose glass slides carrying calcite-PSS crystals were embedded in poly (methyl methacrylate) (PMMA) and subsequently finely polished. The samples were placed into a mould and immersed in a liquid mixture containing methylmethacrylat (MMA, $10 \mathrm{~g}$ ) and AIBN (azodiisobutyronitril, $30 \mathrm{mg}$ ). Subsequently, the specimens were transferred to an oven and kept at a temperature of $40{ }^{\circ} \mathrm{C}$ for one day. Thereafter, the temperature was increased to $48{ }^{\circ} \mathrm{C}$ (holding time 1 day), so that gradual polymerization of the monomer could take place. Finally, the samples were kept for one further day at $55{ }^{\circ} \mathrm{C}$ to allow a complete curing process. After being embedded in PMMA the respective specimens were cut as close as possible above the crystal surface by using a low speed saw (ISOMET, Buehler, Germany). To obtain a sufficiently flat surface, a polishing machine (type 1PM57, Logitech, UK) was applied. For grinding $\mathrm{SiC}$ papers of different grades $(1200,2400$ and 4000) were utilized consecutively. Finally, the samples were polished with $3 \mu \mathrm{m}$ and $1 \mu \mathrm{m}$ diamond papers, respectively.

Biogenic calcite crystals were extracted from the prismatic layer of Pinna nobilis shells by sonication in sodium hypochlorite. ${ }^{[4]]}$ The calcitic prisms were embedded and finely polished parallel to the basal plane of the prisms, in order to indent them in the same direction as the biomimetic calcite-PSS crystals.

The nanoindentation studies were carried out in ambient conditions using an Ubi nanoindentation instrument (Hysitron) with a Berkovich diamond tip. To obtain hardness and reduced modulus all loaddisplacement curves were analyzed using the method described by Oliver and Pharr. ${ }^{[42]} \mathrm{A}$ minimum of 10 indentations with a maximal load of $1500 \mu \mathrm{N}$ was performed on each specimen. A dwell time of $15 \mathrm{~s}$ was applied at peak load to eliminate creep behavior.

\section{Acknowledgements}

The authors would like to thank Chenghao Li, Stefan Siegel, Ivo Zizak and Christoph Gilow for support during synchrotron experiments at the $\mu$-spot beamline (BESSY II, Berlin). They gratefully acknowledge Ingrid Zenke and Birgit Schonert for assistance in laboratory X-ray measurements and Christine Lausser as well as Eric Schönemann for support with sample preparation. The authors are grateful to Frédéric Marin and Emil Zolotoyabko for supplying the biogenic calcite prisms used as reference sample for the nanoindentation experiments. The authors would like to thank Anne Heilig for AFM work and Heike Runge as well as Rona Pitschke for TEM. The European Synchrotron Radiation Facility (Grenoble, France) and the Helmholtz-Zentrum für Materialien und Energie (Berlin, Germany) are acknowledged for the provision of synchrotron facilities. This work was partially funded by the German Research Foundation (DFG) in the framework of priority program SPP 1420 "Biomimetic Materials Research: Functionality by Hierarchical Structuring of Materials".

[1] N. A. J. M. Sommerdijk, G. de With, Chem. Rev. 2008, 108, 4499.

[2] A.-W. Xu, Y. Ma, H. Coelfen, J. Mater. Chem. 2007, 17, 415.

[3] E. Beniash, WIREs Nanomed. Nanobiotechnol. 2011, 3, 47.

[4] S. Mann, Biomineralization. Principles and Concepts in Bio-inorganic Materials Chemistry, Oxford University Press, New York 2001.

[5] A. Berman, L. Addadi, A. Kvick, L. Leiserowitz, M. Nelson, S. Weiner, Science 1990, 250, 664.

[6] A. Berman, L. Addadi, S. Weiner, Nature 1988, 331, 546.

[7] F. Marin, R. Amons, N. Guichard, M. Stigter, A. Hecker, G. Luquet, P. Layrolle, G. Alcaraz, C. Riondet, P. Westbroek, J. Biol. Chem. 2005, 280, 33895

[8] B. Pokroy, J. P. Quintana, E. N. Caspi, A. Berner, E. Zolotoyabko, Nat. Mater. 2004, 3, 900.

[9] B. Pokroy, A. N. Fitch, F. Marin, M. Kapon, N. Adir, E. Zolotoyabko, J. Struct. Biol. 2006, 155, 96.

[10] F. C. Meldrum, H. Coelfen, Chem. Rev. 2008, 108, 4332.

[11] S. Ludwigs, U. Steiner, A. N. Kulak, R. Lam, F. C. Meldrum, Adv. Mater. 2006, 18, 2270.

[12] H. Li, H. L. Xin, D. A. Muller, L. A. Estroff, Science 2009, 326, 1244.

[13] J. Aizenberg, A. J. Black, G. H. Whitesides, J. Am. Chem. Soc. 1999, $121,4500$.

[14] B. Pokroy, J. Aizenberg, CrystEngComm 2007, 9, 1219.

[15] A. N. Kulak, P. Iddon, Y. Li, S. P. Armes, H. Colfen, O. Paris R. M. Wilson, F. C. Meldrum, J. Am. Chem. Soc. 2007, 129, 3729.

[16] T. X. Wang, H. Colfen, M. Antonietti, J. Am. Chem. Soc. 2005, 127, 3246.

[17] L. B. Gower, D. A. Tirrell, J. Cryst. Growth 1998, 191, 153-160.

[18] L. B. Gower, Chem. Rev. 2008, 108, 4551-4627.

[19] K. M. Towe, G. R. Thompson, Calcif. Tissue Res. 1972, 10, 38.

[20] F. Nudelman, H. H. Chen, H. A. Goldberg, S. Weiner, L. Addadi, Farad. Discuss. 2007, 136, 9

[21] F. Nudelman, B. A. Gotliv, L. Addadi, S. Weiner, J. Struct. Biol. 2006 $153,176$.

[22] L. Addadi, J. Moradian, E. Shay, N. G. Maroudas, S. Weiner, Proc. Natl. Acad. Sci. USA 1987, 84, 2732.

[23] H. A. Lowenstam, S. Weiner, On Biomineralization, Oxford University Press, New York, USA 1989.

[24] S. Weiner, P. M. Dove, in Biomineralization, Vol. 54 (Eds: P. M. Dove, J. J. DeYoreo, S. Weiner), The Mineralogical Society of America, Washington, DC 2003.

[25] J. Aizenberg, A. J. Black, G. H. Whitesides, J. Am. Chem. Soc. 1999 $121,4500$.

[26] G. Wulff, Z. Krystallogr. Mineralog. 1901, 34, 449. 
[27] D. M. Paganin, Coherent X-Ray Optics, Oxford University Press, New York 2006.

[28] P. Zaslansky, S. Zabler, P. Fratzl, Dent. Mater. 2010, 26, E1.

[29] O. Paris, C. Li, S. Siegel, G. Weseloh, F. Emmerling, H. Riesemeier, A. Erko, P. Fratzl, J. Appl. Crystallogr. 2007, 40, S466.

[30] Y.-Y. Kim, K. Ganesan, P. Yang, A. N. Kulak, S. Borukhin, S. Pechook, L. Ribeiro, R. Kroeger, S. J. Eichhorn, S. P. Armes, B. Pokroy, F. C. Meldrum, Nat. Mater. 2011, 10, 890.

[31] O. Glatter, O. Kratky, Eds., Small Angle X-Ray Scattering, Academic Press, London 1982.

[32] P. Debye, J. Phys. Colloid Chem. 1947, 51, 18.

[33] S. Ciccariello, J. Goodisman, H. Brumberger, J. Appl.Crystallogr. 1988, 21, 117.
[34] H. D. Bale, P. W. Schmidt, Phys. Rev. Lett. 1984, 53, 596.

[35] C. Gilow, E. Zolotoyabko, O. Paris, P. Fratzl, B. Aichmayer, Cryst. Growth Des. 2011, 11, 2054.

[36] A. N. Fitch, J. Res. Natl. Inst. Stand. 2004, 109, 133.

[37] B. Pokroy, A. N. Fitch, E. Zolotoyabko, Adv. Mater. 2006, 18, 2363.

[38] Y. Ma, S. R. Cohen, L. Addadi, S. Weiner, Adv. Mater. 2008, 20, 1555.

[39] X. Li, Z.-H. Xu, R. Wang, Nano Lett. 2006, 6, 2301.

[40] P. Fratzl, R. Weinkamer, Prog. Mater. Sci. 2007, 52, 1263.

[41] Y. Dauphin, J. P. Cuif, J. Doucet, M. Salome, J. Susini, C. T. Williams, Mar. Biol. 2003, 142, 299.

[42] W. C. Oliver, G. M. Pharr, J. Mater. Res. 1992, 7, 1564. 